\title{
Proteção infantil durante a COVID-19: até quando os casos de maus-tratos infantis continuarão sendo subnotificados?
}

\author{
Child protection during COVID-19: how long will \\ child abuse cases be underreported?
}

\author{
Protección infantil durante la COVID-19: ¿hasta \\ cuándo serán subregistrados los casos de \\ malos tratos infantiles?
}

\author{
Mateus Luz Levandowski 1 \\ Douglas Nunes Stahnke 2 \\ Tiago N. Munhoz 3,4 \\ Jean Von Hohendorff 5 \\ Roberta Salvador-Silva 6
}

doi: 10.1590/0102-311X00078421

Agradecemos as autoras ${ }^{1}$ pelo interesse e comentários sobre o nosso artigo ${ }^{2}$. Consideramos urgente discutir e divulgar amplamente esses resultados para a comunidade em geral, principalmente para as pessoas formadoras de políticas públicas.

Embora nosso artigo tenha demonstrado redução das notificações de maus-tratos na infância no começo da pandemia no Brasil (março e abril de 2020) 2 e mais de um ano tenha se passado desde a coleta dos dados, no momento o Brasil lidera estatísticas de casos e mortes em relação à COVID-19. Até o dia 21 de março de 2021 já foram 2.713.028 mortes por COVID-19 no mundo, sendo 294.042 no Brasil 3. Mesmo o Brasil representando apenas 2,7\% da população global, o país sozinho representa aproximadamente 10\% das mortes por COVID-19 no mundo, sendo que na primeira semana de março de 2021, o Brasil sozinho foi responsável por quase 20\% das mortes por COVID-19 em todo o planeta 4. Até a presente data, a curva de contágios e mortes por COVID-19 segue subindo vertiginosamente, enquanto apenas $1,6 \%$ da população recebeu as duas doses da vacina.

Nesse sentido, ainda é necessário que estados e municípios adotem diversas medidas de distanciamento social e fechamento de serviços não-essenciais para conter a transmissão exponencial do vírus. No Rio Grande do Sul, por exemplo, local onde foi conduzido o nosso estudo, estamos na terceira semana consecutiva (início em 1o de março) em bandeira preta 5 , com diversos estabelecimentos fechados, assim como as escolas ${ }^{6}$. Se sabemos que a maior parte dos atos de violência contra crianças acontece dentro de casa e que são as educadoras e os educadores os maiores responsáveis pela identificação e notificação de casos de maus-tratos 7, é de se esperar que o Rio Grande do Sul, assim como outros estados brasileiros, permaneça com o sério problema de subnotificação de maus-tratos infantis durante todo o ano de 2021. É urgente o poder público elaborar estratégias para mitigar esse impacto.

Além de nosso artigo demonstrando subnotificação de maus-tratos na infância durante o início da pandemia, outros artigos no continente americano encontraram o mesmo. Sete artigos encontraram subnotificação em diferentes estados do Estados Unidos 8,9,10,11,12,13,14, um artigo encontrou subnotificação no México 15 e mais três artigos encontraram subnotificação em outros estados brasileiros (Sergipe 16, Piauí 17 e Santa Catarina 18), além do nosso estudo no Rio Grande do Sul 2. Se por um lado há subnotificação e impossibilidade de proteção e cuidado das crianças e famílias atingidas, estudos recentes vêm demonstrando que, de fato, há aumento de violência nos períodos de maior isolamento e recessão econômica durante a COVID-19 19,20,21.
1 Universidade Federal de Pelotas, Pelotas, Brasil. 2 Universidade do Vale do Rio dos Sinos, São Leopoldo, Brasil.

3 Curso de Psicologia, Universidade Federal de Pelotas, Pelotas, Brasil.

4 Programa de Pós-graduação em Epidemiologia,

Universidade Federal de Pelotas, Pelotas, Brasil.

5 Programa de Pós-graduação em Psicologia, Faculdade Meridional (IMED), Passo Fundo, Brasil.

${ }^{6}$ Human Development and Violence Research Centre, Universidade Federal de Pelotas, Pelotas, Brasil.

\section{Correspondência}

M. L. Levandowski Universidade Federal de Pelotas.

Av. Duque de Caxias 250, Pelotas, RS 96030-000, Brasil. luzlevandowski@gmail.com 
No momento, estamos elaborando um novo artigo para compreender se o impacto identificado em março e abril de 2020 segue a mesma tendência ao longo de 2020 e início de 2021. Nossos dados ainda em análise e não publicados apontam que a subnotificação seguiu ocorrendo de maio a dezembro de 2020 e entre janeiro e fevereiro de 2021. Desta maneira, políticas públicas precisam ser planejadas para prevenir o aumento da violência (e subnotificação) contra crianças e adolescentes durante as sucessivas ondas de distanciamento social.

Por fim, analisando os dados sobre COVID-19 no Brasil, imagina-se que a pandemia irá durar ainda mais tempo em nosso país, sendo urgente que estratégias sejam elaboradas para a proteção desses jovens que não estão tendo a chance de pedido de socorro. Defendemos o uso de máscara e de distanciamento social para barrar o avanço do vírus, que os serviços de proteção a crianças e adolescentes devam pensar em estratégias de visitas domiciliares (tomando todos os cuidados sanitários) ou videoconferências e chamadas telefônicas como instrumentos de cuidado e prevenção, além de que a população seja massivamente vacinada, com as professoras, os professores e demais trabalhadoras e trabalhadores da rede de ensino básico e fundamental na lista de prioridades.

\section{Colaboradores}

M. L. Levandowski, D. N. Stahnke e R. Salvador-Silva participaram da concepção do texto e da coleta, análise e interpretação dos dados. T. N. Munhoz e J. Von Hohendorff colaboraram na interpretação dos dados e na redação e revisão crítica do texto.

\section{Informações adicionais}

ORCID: Mateus Luz Levandowski (0000-00026188-620X); Douglas Nunes Stahnke (0000-00026871-4355); Tiago N. Munhoz (0000-0003-12819542); Jean Von Hohendorff (0000-0002-74145312); Roberta Salvador-Silva (0000-0002-77297928).
1. Hamada AKC, Cassol MEG, Baggio AO, Marcon CEM. Impacto do distanciamento social nas notificações de violência conta crianças e adolescentes no Rio Grande do Sul, Brasil: uma análise consensual. Cad Saúde Pública 2021; 37:e0070521.

2. Levandowski ML, Stahnke DN, Munhoz TN, Von Hohendorff J, Salvador-Silva R. Impacto do distanciamento social nas notificações de violência contra crianças e adolescentes no Rio Grande do Sul, Brasil. Cad Saúde Pública 2021; 37:e00140020.

3. Ministério da Saúde. Painel Coronavírus. https://covid.saude.gov.br/ (acessado em 21/ Mar/2021).

4. Hallal P. É melhor NÃO ir se acostumando. Folha de S.Paulo 2021; 9 mar. https:// www1.folha.uol.com.br/colunas/pedro-hal lal/2021/03/e-melhor-nao-ir-se-acostuman do.shtml.

5. Rio Grande do Sul. Bandeira preta e suspensão da cogestão: governo esclarece dúvidas sobre novas medidas. https://www.estado.rs.gov. $\mathrm{br} /$ bandeira-preta-e-suspensao-da-cogestaogoverno-esclarece-duvidas-sobre-novas-me didas (acessado em 20/Mar/2021).

6. Juíza suspende aulas presenciais em escolas públicas e privadas do RS. GZH 2021; 1 mar. https://gauchazh.clicrbs.com.br/coronavirusservico/noticia/2021/03/juiza-suspende-au las-presenciais-em-escolas-publicas-e-priva das-do-rs-cklq05sk8003z014nxvwqu08e.html. 
7. Children's Bureau, U.S. Department of Health and Human Services. Child maltreatment 2018. https://www.acf.hhs.gov/sites/default/ files/documents/cb/cm2018.pdf (acessado em 21/Mar/2021).

8. Barboza GE, Schiamberg LB, Pachl L. A spatiotemporal analysis of the impact of COVID-19 on child abuse and neglect in the city of Los Angeles. Child Abuse Negl 2020; [Epub ahead of print].

9. Baron EJ, Goldstein EG, Wallace CT. Suffering in silence: how COVID-19 school closures inhibit the reporting of child maltreatment. J Public Econ 2020; 190:104258.

10. McLay MM. When "shelter-in-place" isn't shelter that's safe: a rapid analysis of domestic violence case differences during the COVID-19 pandemic and stay-at-home orders. medRxiv 2020; 5 nov. https://www.me drxiv.org/content/10.1101/2020.05.29.20117 $366 v 3$.

11. Musser ED, Riopelle C, Latham R. Child maltreatment in the time of COVID-19: changes in the Florida foster care system surrounding the COVID-19 safer-at-home order. Child Abus Negl 2021; [Epub ahead of print].

12. Rapoport E, Reisert H, Schoeman E, Adesman A. Reporting of child maltreatment during the SARS-CoV-2 pandemic in New York City from March to May 2020. Child Abus Negl 2020; [Epub ahead of print].

13. Whaling K, Der Sarkissian A, Larez N, Sharkey JD, Allen MA, Nylund-Gibson K. Reduced child maltreatment prevention service case openings during COVID-19. Research Square 2020; 23 mai. https://www.researchsquare. com/article/rs-30930/v1.

14. Whelan J, Hartwell M, Chesher T, Coffey S, Hendrix AD, Passmore SJ, et al. Deviations in criminal filings of child abuse and neglect during COVID-19 from forecasted models: an analysis of the state of Oklahoma, USA. Child Abus Negl 2020; [Epub ahead of print].
15. Cabrera-Hernández F, Padilla-Romo M. Hidden violence: how COVID-19 school closures reduced the reporting of child maltreatment. Latin American Economic Review 2020; 29:4.

16. Martins-Filho PR, Damascena NP, Lage RCM, Sposato KB. Decrease in child abuse notifications during COVID-19 outbreak: a reason for worry or celebration? J Paediatr Child Health 2020; 56:1980-1.

17. Trajano RKN, Lyra CVV, Sá TYG, Gomes ACA. Comparativo de casos de violência sexual contra criança e adolescente no período 2018-2020. Research, Society and Development 2021; 10:e11710111384.

18. Platt VB, Guedert JM, Coelho EBS. Violência contra crianças e adolescentes: notificações e alerta em tempos de pandemia. Rev Paul Pediatr 2021; 39:e2020267.

19. Calvano C, Engelke L, Di Bella J, Kindermann J, Renneberg B, Winter SM. Families in the COVID-19 pandemic: parental stress, parent mental health and the occurrence of adverse childhood experiences-results of a representative survey in Germany. Eur Child Adolesc Psychiatry 2021; [Online ahead of print].

20. Jeharsae R, Jae-noh M, Jae-a-lee H, Waeteh S, Nimu N, Chewae C, et al. Associations between stress and child verbal abuse and corporal punishment during the COVID-19 pandemic and potential effect modification by lockdown measures. medRxiv 2021; 6 jan. https://medrxiv.org/cgi/content/ short/2021.01.05.20248973.

21. Lawson M, Piel MH, Simon M. Child maltreatment during the COVID-19 pandemic: consequences of parental job loss on psychological and physical abuse towards children. Child Abus Negl 2020; 110:104709.

Recebido em 23/Mar/2021

Aprovado em 25/Mar/2021 